\title{
Existence and iterative approximation for generalized equilibrium problems for a countable family of nonexpansive mappings in banach spaces
}

Uthai Kamraksa $^{1}$ and Rabian Wangkeeree ${ }^{1,2^{*}}$

* Correspondence: uthaikam@hotmail.com

'Department of Mathematics, Faculty of Science, Naresuan University, Phitsanulok 65000, Thailand

Full list of author information is available at the end of the article

\begin{abstract}
We first prove the existence of a solution of the generalized equilibrium problem (GEP) using the KKM mapping in a Banach space setting. Then, by virtue of this result, we construct a hybrid algorithm for finding a common element in the solution set of a GEP and the fixed point set of countable family of nonexpansive mappings in the frameworks of Banach spaces. By means of a projection technique, we also prove that the sequences generated by the hybrid algorithm converge strongly to a common element in the solution set of GEP and common fixed point set of nonexpansive mappings.

AMS Subject Classification: 47H09, 47H10

Keywords: Banach space, Fixed point, Metric projection, Generalized equilibrium problem, Nonexpansive mapping
\end{abstract}

\section{Introduction}

Let $E$ be a real Banach space with the dual $E^{*}$ and $C$ be a nonempty closed convex subset of $E$. We denote by $\mathcal{N}$ and $\mathcal{R}$ the sets of positive integers and real numbers, respectively. Also, we denote by $J$ the normalized duality mapping from $E$ to $2^{E^{*}}$ defined by

$$
J x=\left\{x^{*} \in E^{*}:\left\langle x, x^{*}\right\rangle=\|x\|^{2}=\left\|x^{*}\right\|^{2}\right\}, \quad \forall x \in E,
$$

where $\langle\cdot\rangle$,$\rangle denotes the generalized duality pairing. We know that if E$ is smooth, then $J$ is single-valued and if $E$ is uniformly smooth, then $J$ is uniformly norm-to-norm continuous on bounded subsets of $E$. We shall still denote by $J$ the single-valued duality mapping. Let $f: C \times C \rightarrow \mathcal{R}$ be a bifunction and $A: C \rightarrow E^{*}$ be a nonlinear mapping. We consider the following generalized equilibrium problem (GEP):

$$
\text { Find } u \in C \text { such that } f(u, y)+\langle A u, y-u\rangle \geq 0, \quad \forall y \in C \text {. }
$$

The set of such $u \in C$ is denoted by GEP (f), i.e.,

$$
\operatorname{GEP}(f)=\{u \in C: f(u, y)+\langle A u, y-u\rangle \geq 0, \quad \forall y \in C\} .
$$

(c) 2011 Kamraksa and Wangkeeree; licensee Springer. This is an Open Access article distributed under the terms of the Creative Commons Attribution License (http://creativecommons.org/licenses/by/2.0), which permits unrestricted use, distribution, and reproduction in any medium, provided the original work is properly cited. 
Whenever $E=H$ a Hilbert space, the problem (1.1) was introduced and studied by Takahashi and Takahashi [1]. Similar problems have been studied extensively recently. In the case of $A \equiv 0, G E P(f)$ is denoted by $E P(f)$. In the case of $f \equiv 0, E P$ is also denoted by $\operatorname{VI}(C, A)$. Problem (1.1) is very general in the sense that it includes, as spacial cases, optimization problems, variational inequalities, minimax problems, the Nash equilibrium problem in noncooperative games, and others; see, e.g., [2,3]. A mapping $T: C \rightarrow E$ is called nonexpansive if $\|T x-T y\| \leq\|x-y\|$ for all $x, y \in C$. Denote by $F(T)$ the set of fixed points of $T$, that is, $F(T)=\{x \in C$ : $T x=x\}$. A mapping $A: C \rightarrow E^{*}$ is called $\alpha$-inverse-strongly monotone, if there exists an $\alpha>0$ such that

$$
\langle A x-A y, x-y\rangle \geq \alpha\|A x-A y\|^{2}, \quad \forall x, y \in C .
$$

It is easy to see that if $A: C \rightarrow E^{*}$ is an $\alpha$-inverse-strongly monotone mapping, then it is $1 / \alpha$ - Lipschitzian.

In 1953, Mann [4] introduced the following iterative procedure to approximate a fixed point of a nonexpansive mapping $T$ in a Hilbert space $H$ :

$$
x_{n+1}=\alpha_{n} x_{n}+\left(1-\alpha_{n}\right) T x_{n}, \quad \forall n \in \mathcal{N},
$$

where the initial point $x_{0}$ is taken in $C$ arbitrarily and $\left\{\alpha_{n}\right\}$ is a sequence in $[0,1]$.

However, we note that Manns iteration process (1.2) has only weak convergence, in general; for instance, see [5-7].

Let $C$ be a nonempty, closed, and convex subset of a Banach space $E$ and $\left\{T_{n}\right\}$ be sequence of mappings of $C$ into itself such that $\bigcap_{n=1}^{\infty} F\left(T_{n}\right) \neq \emptyset$. Then, $\left\{T_{n}\right\}$ is said to satisfy the NST-condition if for each bounded sequence $\left\{z_{n}\right\} \subset C$,

$$
\lim _{n \rightarrow \infty}\left\|z_{n}-T_{n} z_{n}\right\|=0
$$

implies $\omega_{w}\left(z_{n}\right) \subset \bigcap_{n=1}^{\infty} F\left(T_{n}\right)$, where $\omega_{w}\left(z_{n}\right)$ is the set of all weak cluster points of $\left\{z_{n}\right\}$; see $[8-10]$.

In 2008, Takahashi et al. [11] has adapted Nakajo and Takahashi's [12] idea to modify the process (1.2) so that strong convergence has been guaranteed. They proposed the following modification for a family of nonexpansive mappings in a Hilbert space: $x_{0} \in H, C_{1}=C, u_{1}=P_{C_{1}} x_{0}$ and

$$
\left\{\begin{array}{l}
y_{n}=\alpha_{n} u_{n}+\left(1-\alpha_{n}\right) T_{n} u_{n} \\
C_{n+1}=\left\{z \in C_{n}:\left\|y_{n}-z\right\| \leq\left\|u_{n}-z\right\|\right\} \\
u_{n+1}=P_{C_{n+1}} x_{0}, \quad n \in \mathcal{N}
\end{array}\right.
$$

where $0 \leq \alpha_{n} \leq a<1$ for all $n \in \mathcal{N}$. They proved that if $\left\{T_{n}\right\}$ satisfies the NSTcondition, then $\left\{u_{n}\right\}$ generated by (1.3) converges strongly to a common fixed point of $T_{n}$.

Recently, motivated by Nakajo and Takahashi [12] and Xu [13], Matsushita and Takahashi [14] introduced the iterative algorithm for finding fixed points of nonexpansive mappings in a uniformly convex and smooth Banach space: $x_{0}=x \in C$ and

$$
\left\{\begin{array}{l}
C_{n}=\overline{c o}\left\{z \in C:\|z-T z\| \leq t_{n}\left\|x_{n}-T x_{n}\right\|\right\}, \\
D_{n}=\left\{z \in C:\left\langle x_{n}-z, J\left(x-x_{n}\right)\right\rangle \geq 0\right\}, \\
x_{n+1}=P_{C_{n} \cap D_{n}} x, \quad n \geq 0,
\end{array}\right.
$$


where $\overline{c o} D$ denotes the convex closure of the set $D,\left\{t_{n}\right\}$ is a sequence in $(0,1)$ with $t_{n}$ $\rightarrow 0$, and $P_{C_{n} \cap D_{n}}$ is the metric projection from $E$ onto $C_{n} \cap D_{n}$. They proved that $\left\{x_{n}\right\}$ generated by (1.4) converges strongly to a fixed point of $T$.

Very recently, Kimura and Nakajo [15] investigated iterative schemes for finding common fixed points of a family of nonexpansive mappings and proved strong convergence theorems by using the Mosco convergence technique in a uniformly convex and smooth Banach space. In particular, they proposed the following algorithm: $x_{1}=x \in C$ and

$$
\left\{\begin{array}{l}
C_{n}=\overline{c o}\left\{z \in C:\left\|z-T_{n} z\right\| \leq t_{n}\left\|x_{n}-T_{n} x_{n}\right\|\right\}, \\
D_{n}=\left\{z \in C:\left\langle x_{n}-z, J\left(x-x_{n}\right)\right\rangle \geq 0\right\}, \\
x_{n+1}=P_{C_{n} \cap D_{n}} x, \quad n \geq 0,
\end{array}\right.
$$

where $\left\{t_{n}\right\}$ is a sequence in $(0,1)$ with $t_{n} \rightarrow 0$ as $n \rightarrow \infty$. They proved that if $\left\{T_{n}\right\}$ satisfies the NST-condition, then $\left\{x_{n}\right\}$ converges strongly to a common fixed point of $T_{n}$.

Motivated and inspired by Nakajo and Takahashi [12], Takahashi et al. [11], Xu [13], Masushita and Takahashi [14], and Kimura and Nakajo [15], we introduce a hybrid projection algorithm for finding a common element in the solution set of a GEP and the common fixed point set of a family of nonexpansive mappings in a Banach space setting.

\section{Preliminaries}

Let $E$ be a real Banach space and let $U=\{x \in E:\|x\|=1\}$ be the unit sphere of $E$.

A Banach space $E$ is said to be strictly convex if for any $x, y \in U$,

$$
x \neq y \text { implies }-|x+y| \mid<2 .
$$

It is also said to be uniformly convex if for each $\varepsilon \in(0,2]$, there exists $\delta>0$ such that for any $x, y \in U$,

$$
\| x-y|| \geq \varepsilon \text { implies }-|x+y| \mid<2(1-\delta) .
$$

It is known that a uniformly convex Banach space is reflexive and strictly convex. Define a function $\delta:[0,2] \rightarrow[0,1]$ called the modulus of convexity of $E$ as follows:

$$
\delta(\varepsilon)=\inf \left\{1-\left\|\frac{x+y}{2}\right\|: x, y \in E,\|x\|=\|y\|=1,\|x-y\| \geq \varepsilon\right\} .
$$

Then, $E$ is uniformly convex if and only if $\delta(\varepsilon)>0$ for all $\varepsilon \in(0,2]$. A Banach space $E$ is said to be smooth if the limit

$$
\lim _{t \rightarrow 0} \frac{\|x+t y\|-\|x\|}{t}
$$

exists for all $x, y \in U$. Let $C$ be a nonempty, closed, and convex subset of a reflexive, strictly convex and smooth Banach space $E$. Then, for any $x \in E$, there exists a unique point $x_{0} \in C$ such that

$$
\left\|x_{0}-x\right\| \leq \min _{y \in C}\|y-x\| .
$$

The mapping $P_{C}: E \rightarrow C$ defined by $P_{C} \times=x_{0}$ is called the metric projection from $E$ onto $C$. Let $x \in E$ and $u \in C$. Then, it is known that $u=P_{C} \times$ if and only if

$$
\left\langle u-y_{1} J(x-u)\right\rangle \geq 0
$$


for all $y \in C$; see [16] for more details. It is well known that if $P_{C}$ is a metric projection from a real Hilbert space $H$ onto a nonempty, closed, and convex subset $C$, then $P_{C}$ is nonexpansive. However, in a general Banach space, this fact is not true.

In the sequel, we will need the following lemmas.

Lemma 2.1. [17]Let $E$ be a uniformly convex Banach space, $\left\{\alpha_{n}\right\}$ be a sequence of real numbers such that $0<b \leq \alpha_{n} \leq c<1$ for all $n \geq 1$, and $\left\{x_{n}\right\}$ and $\left\{y_{n}\right\}$ be sequences in $E$ such that $\lim \sup _{n \rightarrow \infty}|| x_{n}\left\|\leq d, \lim \sup _{n \rightarrow \infty}|| y_{n}\right\| \leq d$ and $\lim _{n \rightarrow \infty}|| \alpha_{n} x_{n}+\left(1-\alpha_{n}\right)$ $y_{n} \|=d$. Then, $\lim _{n \rightarrow \infty}|| x_{n}-y_{n} \|=0$.

Lemma 2.2. [18]Let $C$ be a bounded, closed, and convex subset of a uniformly convex Banach space E. Then, there exists a strictly increasing, convex, and continuous function $\gamma:[0, \infty) \rightarrow[0, \infty)$ such that $\gamma(0)=0$ and

$$
\gamma\left(\left\|T\left(\sum_{i=1}^{n} \lambda_{i} x_{i}\right)-\sum_{i=1}^{n} \lambda_{i} T x_{i}\right\|\right) \leq \max _{1 \leq j \leq k \leq n}\left(\left\|x_{j}-x_{k}\right\|-\left\|T x_{j}-T x_{k}\right\|\right)
$$

for all $n \in \mathcal{N},\left\{x_{1}, x_{2}, \ldots, x_{n}\right\} \subset C,\left\{\lambda_{1}, \lambda_{2}, \ldots, \lambda_{n}\right\} \subset[0,1]$ with $\sum_{i=1}^{n} \lambda_{i}=1$ and nonexpansive mapping $T$ of $C$ into $E$.

Following Bruck's [19] idea, we know the following result for a convex combination of nonexpansive mappings which is considered by Aoyama et al. [20] and Kimura and Nakajo [15].

Lemma 2.3. [15] Let $C$ be a nonempty, closed, and convex subset of a uniformly convex Banach space $E$ and $\left\{S_{n}\right\}$ be a family of nonexpansive mappings of $C$ into itself such that $F=\bigcap_{n=1}^{\infty} F\left(S_{n}\right) \neq \emptyset$. Let $\left\{\beta_{n}^{k}\right\}$ be a family of nonnegative numbers with indices $n, k \in \mathcal{N}$ with $k \leq n$ such that

(i) $\sum_{k=1}^{n} \beta_{n}^{k}=1$ for every $n \in \mathcal{N}$;

(ii) $\lim _{n \rightarrow \infty} \beta_{n}^{k}>$ ofor every $k \in \mathcal{N}$

and let $T_{n}=\alpha_{n} I+\left(1-\alpha_{n}\right) \sum_{k=1}^{n} \beta_{n}^{k} S_{k}$ for all $n \in \mathcal{N}$, where $\left\{\alpha_{n}\right\} \subset[a, b]$ for some $a, b$ $\in(0,1)$ with $a \leq b$. Then, $\left\{T_{n}\right\}$ is a family of nonexpansive mappings of $C$ into itself with $\bigcap_{n=1}^{\infty} F\left(T_{n}\right)=$ Fand satisfies the NST-condition.

Now, let us turn to following well-known concept and result.

Definition 2.4. Let $B$ be a subset of topological vector space $X$. A mapping $G: B \rightarrow 2^{X}$ is called a KKM mapping if $c o\left\{x_{1}, x_{2}, \ldots, x_{m}\right\} \subset \bigcup_{i=1}^{m} G\left(x_{i}\right)$ for $x_{i} \in B$ and $i=1,2, \ldots, m$, where $c o A$ denotes the convex hull of the set $A$.

Lemma 2.5. [21]Let $B$ be a nonempty subset of a Hausdorff topological vector space $\times$ and let $G: B \rightarrow 2^{X}$ be a KKM mapping. If $G(x)$ is closed for all $x \in B$ and is compact for at least one $x \in B$, then $\cap_{x \in B} G(x) \neq \varnothing$.

\section{Existence results of gep}

Motivated by Takahashi and Zembayashi [22], and Ceng and Yao [23], we next prove the following crucial lemma concerning the GEP in a strictly convex, reflexive, and smooth Banach space. 
Theorem 3.1. Let $C$ be a nonempty, bounded, closed, and convex subset of a smooth, strictly convex, and reflexive Banach space $E$, let $f$ be a bifunction from $C \times C$ to Rsatisfying (A1)-(A4), where

(A1) $f(x, x)=0$ for all $x \in C$;

(A2) $f$ is monotone, i.e. $f(x, y)+f(y, x) \leq 0$ for all $x, y \in C$;

(A3) for all $y \in C, f(., y)$ is weakly upper semicontinuous;

(A4) for all $x \in C, f(x,$.$) is convex.$

Let $A$ be $\alpha$-inverse strongly monotone of $C$ into $E^{*}$. For all $r>0$ and $x \in E$, define the mapping $S_{r}: E \rightarrow 2^{C}$ as follows:

$$
S_{r}(x)=\left\{z \in C: f(z, y)+\langle A z, y-z\rangle+\frac{1}{r}\langle y-z, J(z-x)\rangle \geq 0, \quad \forall y \in C\right\} .
$$

Then, the following statements hold:

(1) for each $x \in E, S_{r}(x) \neq \varnothing$;

(2) $S_{r}$ is single-valued;

(3) $\left\langle S_{r}(x)-S_{r}(y), J\left(S_{r} x-x\right)\right\rangle \leq\left\langle S_{r}(x)-S_{r}(y), J\left(S_{r} y-y\right)\right\rangle$ for all $x, y \in E$;

(4) $F\left(S_{r}\right)=G E P(f)$;

(5) GEP(f) is nonempty, closed, and convex.

Proof. (1) Let $x_{0}$ be any given point in $E$. For each $y \in C$, we define the mapping $G$ : $C \rightarrow 2^{E}$ by

$$
G(y)=\left\{z \in C: f(z, y)+\langle A z, y-z\rangle+\frac{1}{r}\left\langle y-z, J\left(z-x_{0}\right)\right\rangle \geq 0\right\} \quad \text { for all } y \in C .
$$

It is easily seen that $y \in G(y)$, and hence $G(y) . \neq \varnothing$

(a) First, we will show that $G$ is a KKM mapping. Suppose that there exists a finite subset $\left\{y_{1}, y_{2}, \ldots, y_{m}\right\}$ of $C$ and $\alpha_{i}>0$ with $\sum_{i=1}^{m} \alpha_{i}=1$ such that $\hat{x}=\sum_{i=1}^{m} \alpha_{i} y_{i} \notin G\left(y_{i}\right)$ for all $i=1,2, \ldots, m$. It follows that

$$
f\left(\hat{x}, y_{i}\right)+\left\langle A \hat{x}, y_{i}-\hat{x}\right\rangle+\frac{1}{r}\left\langle y_{i}-\hat{x}, J\left(\hat{x}-x_{0}\right)\right\rangle<0, \text { for all } i=1,2, \ldots, m .
$$

By (A1) and (A4), we have

$$
\begin{aligned}
0 & =f(\hat{x}, \hat{x})+\langle A \hat{x}, \hat{x}-\hat{x}\rangle+\frac{1}{r}\left\langle\hat{x}-\hat{x}, J\left(\hat{x}-x_{0}\right)\right\rangle \\
& \leq \sum_{i=1}^{m}\left(f\left(\hat{x}, y_{i}\right)+\left\langle A \hat{x}, y_{i}-\hat{x}\right\rangle+\frac{1}{r}\left\langle y_{i}-\hat{x}, J\left(\hat{x}-x_{0}\right)\right\rangle\right)<0,
\end{aligned}
$$

which is a contradiction. Thus, $G$ is a KKM mapping on $C$.

(b) Next, we show that $G(y)$ is closed for all $y \in C$. Let $\left\{z_{n}\right\}$ be a sequence in $G(y)$ such that $z_{n} \rightarrow z$ as $n \rightarrow \infty$. It then follows from $z_{n} \in G(y)$ that,

$$
f\left(z_{n}, y\right)+\left\langle A z_{n}, y-z_{n}\right\rangle+\frac{1}{r}\left\langle y-z_{n}, J\left(z_{n}-x\right)\right\rangle \geq 0 .
$$


By (A3), the continuity of $J$, and the lower semicontinuity of $\|\cdot\|^{2}$, we obtain from (3.2) that

$$
\begin{aligned}
0 & \leq \liminf _{n \rightarrow \infty}\left[f\left(z_{n}, y\right)+\left\langle A z_{n}, y-z_{n}\right\rangle+\frac{1}{r}\left\langle y-z_{n}, J\left(z_{n}-x_{0}\right)\right\rangle\right] \\
& \leq \limsup _{n \rightarrow \infty}\left[f\left(z_{n}, y\right)+\left\langle A z_{n}, y-z_{n}\right\rangle+\frac{1}{r}\left\langle y-x_{0}, J\left(z_{n}-x_{0}\right)\right\rangle+\frac{1}{r}\left\langle x_{0}-z_{n}, J\left(z_{n}-x_{0}\right)\right\rangle\right] \\
& =\limsup _{n \rightarrow \infty}\left[f\left(z_{n}, y\right)+\left\langle A z_{n}, y-z_{n}\right\rangle+\frac{1}{r}\left\langle y-x_{0}, J\left(z_{n}-x_{0}\right)\right\rangle-\frac{1}{r}\left\|z_{n}-x_{0}\right\|^{2}\right] \\
& \leq \limsup _{n \rightarrow \infty} f\left(z_{n}, y\right)+\limsup _{n \rightarrow \infty}\left\langle A z_{n}, y-z_{n}\right\rangle+\frac{1}{r} \limsup _{n \rightarrow \infty}\left\langle y-x_{0}, J\left(z_{n}-x_{0}\right)\right\rangle-\frac{1}{r} \liminf _{n \rightarrow \infty}\left\|z_{n}-x_{0}\right\|^{2} \\
& \leq f(z, y)+\langle A z, y-z\rangle+\frac{1}{r}\left\langle y-x_{0}, J\left(z-x_{0}\right)\right\rangle-\frac{1}{r}\left\|z-x_{0}\right\|^{2} \\
& =f(z, y)+\langle A z, y-z\rangle+\frac{1}{r}\left\langle y-x_{0}, J\left(z-x_{0}\right)\right\rangle-\frac{1}{r}\left\langle z-x_{0}, J\left(z-x_{0}\right)\right\rangle \\
& =f(z, y)+\langle A z, y-z\rangle+\frac{1}{r}\left\langle y-z_{,} J\left(z-x_{0}\right)\right\rangle .
\end{aligned}
$$

This shows that $z \in G(y)$, and hence $G(y)$ is closed for all $y \in C$.

(c) We prove that $G(y)$ is weakly compact. We now equip $E$ with the weak topology. Then, $C$, as closed, bounded convex subset in a reflexive space, is weakly compact. Hence, $G(y)$ is also weakly compact.

Using (a), (b), and (c) and Lemma 2.5 , we have $\cap_{x \in C} G(y) \neq \varnothing$. It is easily seen that

$$
S_{r}\left(x_{0}\right)=\bigcap_{\gamma \in C} G(\gamma)
$$

Hence, $s_{r}\left(x_{0}\right) \neq \varnothing$. Since $x_{0}$ is arbitrary, we can conclude that $s_{r}(x) \neq \varnothing$ for all $x \in E$.

(2) We prove that $S_{r}$ is single-valued. In fact, for $x \in C$ and $r>0$, let $z_{1}, z_{2} \in S_{r}(x)$. Then,

$$
f\left(z_{1}, z_{2}\right)+\left\langle A z_{1}, z_{2}-z_{1}\right\rangle+\frac{1}{r}\left\langle z_{2}-z_{1}, J\left(z_{1}-x\right)\right\rangle \geq 0 .
$$

and

$$
f\left(z_{2}, z_{1}\right)+\left\langle A z_{2}, z_{1}-z_{2}\right\rangle+\frac{1}{r}\left\langle z_{1}-z_{2}, J\left(z_{2}-x\right)\right\rangle \geq 0 .
$$

Adding the two inequalities and from the condition (A2) and monotonicity of $A$, we have

$$
\begin{aligned}
0 & \leq f\left(z_{1}, z_{2}\right)+f\left(z_{2}, z_{1}\right)+\left\langle A z_{1}, z_{2}-z_{1}\right\rangle+\left\langle A z_{2}, z_{1}-z_{2}\right\rangle+\frac{1}{r}\left\langle z_{2}-z_{1}, J\left(z_{1}-x\right)-J\left(z_{2}-x\right)\right\rangle \\
& \leq\left\langle A z_{1}-A z_{2}, z_{2}-z_{1}\right\rangle+\frac{1}{r}\left\langle z_{2}-z_{1}, J\left(z_{1}-x\right)-J\left(z_{2}-x\right)\right\rangle \\
& \leq-\alpha\left\|A z_{1}-A z_{2}\right\|^{2}+\frac{1}{r}\left\langle z_{2}-z_{1}, J\left(z_{1}-x\right)-J\left(z_{2}-x\right)\right\rangle \\
& \leq \frac{1}{r}\left\langle z_{2}-z_{1}, J\left(z_{1}-x\right)-J\left(z_{2}-x\right)\right\rangle
\end{aligned}
$$

and hence,

$$
\left\langle z_{2}-z_{1} J\left(z_{1}-x\right)-J\left(z_{2}-x\right)\right\rangle \geq 0 .
$$

Hence,

$$
0 \leq\left\langle z_{2}-z_{1}, J\left(z_{1}-x\right)-J\left(z_{2}-x\right)\right\rangle=\left\langle\left(z_{2}-x\right)-\left(z_{1}-x\right), J\left(z_{1}-x\right)-J\left(z_{2}-x\right)\right\rangle .
$$


Since $J$ is monotone and $E$ is strictly convex, we obtain that $z_{1}-x=z_{2}-x$ and hence $z_{1}=z_{2}$.

Therefore $S_{r}$ is single-valued.

(3) For $x, y \in C$, we have

$$
f\left(S_{r} x, S_{r} y\right)+\left\langle A S_{r} x, S_{r} y-S_{r} x\right\rangle+\frac{1}{r}\left\langle S_{r} y-S_{r} x, J\left(S_{r} x-x\right)\right\rangle \geq 0
$$

and

$$
f\left(S_{r} y, S_{r} x\right)+\left\langle A S_{r} y, S_{r} x-S_{r} y\right\rangle+\frac{1}{r}\left\langle S_{r} x-S_{r} y, J\left(S_{r} y-y\right)\right\rangle \geq 0 .
$$

Again, adding the two inequalities, we also have

$$
\left\langle A S_{r} x-A S_{r} y, S_{r} y-S_{r} x\right\rangle+\left\langle S_{r} y-S_{r} x, J\left(S_{r} x-x\right)-J\left(S_{r} y-y\right)\right\rangle \geq 0 .
$$

It follows from monotonicity of $A$ that

$$
\left\langle S_{r} y-S_{r} x, J\left(S_{r} x-x\right)\right\rangle \leq\left\langle S_{r} y-S_{r} x, J\left(S_{r} y-y\right)\right\rangle .
$$

(4) It is easy to see that

$$
\begin{aligned}
z \in F\left(S_{r}\right) & \Leftrightarrow z=S_{r} z \\
& \Leftrightarrow f(z, y)+\langle A z, y-z\rangle+\frac{1}{r}\langle y-z, J(z-z)\rangle \geq 0, \quad \forall y \in C \\
& \Leftrightarrow f(z, y)+\langle A z, y-z\rangle \geq 0, \quad \forall y \in C \\
& \Leftrightarrow z \in G E P(f) .
\end{aligned}
$$

Hence, $F\left(S_{r}\right)=G E P(f)$.

(5) Finally, we claim that $G E P(f)$ is nonempty, closed, and convex. For each $y \in C$, we define the mapping $\Theta: C \rightarrow 2^{E}$ by

$$
\Theta(y)=\{x \in C: f(x, y)+\langle A x, y-x\rangle \geq 0\} .
$$

Since $y \in \Theta(y)$, we have $\Theta(\mathrm{y}) \neq \varnothing$ We prove that $\Theta$ is a KKM mapping on $C$. Suppose that there exists a finite subset $\left\{z_{1}, z_{2}, \ldots, z_{m}\right\}$ of $C$ and $\alpha_{i}>0$ with $\sum_{i=1}^{m} \alpha_{i}=1$ such that $\hat{z}=\sum_{i=1}^{m} \alpha_{i} z_{i} \notin \Theta\left(z_{i}\right)$ for all $i=1,2, \ldots, m$. Then,

$$
f\left(\hat{z}, z_{i}\right)+\left\langle A \hat{z}, z_{i}-\hat{z}\right\rangle<0, \quad i=1,2, \ldots, m .
$$

From (A1) and (A4), we have

$$
0=f(\hat{z}, \hat{z})+\langle A \hat{z}, \hat{z}-\hat{z}\rangle \leq \sum_{i=1}^{m} \alpha_{i}\left(f\left(\hat{z}, z_{i}\right)+\left\langle A \hat{z}, z_{i}-\hat{z}\right\rangle\right)<0,
$$

which is a contradiction. Thus, $\Theta$ is a KKM mapping on $C$.

Next, we prove that $\Theta(y)$ is closed for each $y \in C$. For any $y \in C$, let $\left\{x_{n}\right\}$ be any sequence in $\Theta(y)$ such that $x_{n} \rightarrow x_{0}$. We claim that $x_{0} \in \Theta(y)$. Then, for each $y \in C$, we have

$$
f\left(x_{n}, y\right)+\left\langle A x_{n}, y-x_{n}\right\rangle \geq 0 .
$$

By (A3), we see that

$$
f\left(x_{0}, y\right)+\left\langle A x_{0}, y-x_{0}\right\rangle \geq \limsup _{n \rightarrow \infty} f\left(x_{n}, y\right)+\lim _{n \rightarrow \infty}\left\langle A x_{n}, y-x_{n}\right\rangle \geq 0 .
$$


This shows that $x_{0} \in \Theta(y)$ and $\Theta(y)$ is closed for each $y \in C$. Thus, $\bigcap_{y \in C} \Theta(\gamma)=G E P(f)$ is also closed.

We observe that $\Theta(y)$ is weakly compact. In fact, since $C$ is bounded, closed, and convex, we also have $\Theta(y)$ is weakly compact in the weak topology. By Lemma 2.5, we can conclude that $\bigcap_{y \in C} \Theta(\gamma)=G E P(f) \neq \emptyset$.

Finally, we prove that GEP $(f)$ is convex. In fact, let $u, v \in F\left(S_{r}\right)$ and $z_{t}=t u+(1-t) v$ for $t \in(0,1)$. From (3), we know that

$$
\left\langle S_{r} u-S_{r} z_{t}, J\left(S_{r} z_{t}-z_{t}\right)-J\left(S_{r} u-u\right)\right\rangle \geq 0 .
$$

This yields that

$$
\left\langle u-S_{r} z_{t} J\left(S_{r} z_{t}-z_{t}\right)\right\rangle \geq 0 .
$$

Similarly, we also have

$$
\left\langle v-S_{r} z_{t} J\left(S_{r} z_{t}-z_{t}\right)\right\rangle \geq 0 .
$$

It follows from (3.4) and (3.5) that

$$
\begin{aligned}
\left\|z_{t}-S_{r} z_{t}\right\|^{2} & =\left\langle z_{t}-S_{r} z_{t} J\left(z_{t}-S_{r} z_{t}\right)\right\rangle \\
& =t\left\langle u-S_{r} z_{t} J\left(z_{t}-S_{r} z_{t}\right)\right\rangle+(1-t)\left\langle v-S_{r} z_{t} J\left(z_{t}-S_{r} z_{t}\right)\right\rangle \\
& \leq 0
\end{aligned}
$$

Hence, $z_{t} \in F\left(S_{r}\right)=\operatorname{GEP}(f)$ and hence GEP $(f)$ is convex. This completes the proof.

\section{Strong convergence theorem}

In this section, we prove a strong convergence theorem using a hybrid projection algorithm in a uniformly convex and smooth Banach space.

Theorem 4.1. Let $E$ be a uniformly convex and smooth Banach space and $C$ be a nonempty, bounded, closed, and convex subset of $E$. Let $f$ be a bifunction from $C \times C$ to Rsatisfying (A1)-(A4), $A$ an $\alpha$-inverse strongly monotone mapping of $C$ into $E^{*}$ and $\left\{T_{n}\right\}_{n=0}^{\infty} a$ sequence of nonexpansive mappings of $C$ into itself such that $\Omega:=\bigcap_{n=0}^{\infty} F\left(T_{n}\right) \cap G E P(f) \neq \emptyset$ and suppose that $\left\{T_{n}\right\}_{n=0}^{\infty}$ satisfies the NST-condition. Let $\left\{x_{n}\right\}$ be the sequence in $C$ generated by

$$
\left\{\begin{array}{l}
x_{0} \in C, D_{0}=C, \\
C_{n}=\overline{c o}\left\{z \in C:\left\|z-T_{n} z\right\| \leq t_{n}\left\|x_{n}-T_{n} x_{n}\right\|\right\}, \quad n \geq 1, \\
u_{n} \in C \text { such that } f\left(u_{n}, y\right)+\left\langle A u_{n}, y-u_{n}\right\rangle+\frac{1}{r_{n}}\left\langle y-u_{n} J\left(u_{n}-x_{n}\right)\right\rangle \geq 0, \quad \forall y \in C, n \geq 0, \\
D_{n}=\left\{z \in D_{n-1}:\left\langle u_{n}-z_{1} J\left(x_{n}-u_{n}\right)\right\rangle \geq 0\right\}, \quad n \geq 1, \\
x_{n+1}=P_{C_{n} \cap D_{n}} x_{0}, \quad n \geq 0,
\end{array}\right.
$$

where $\left\{t_{n}\right\}$ and $\left\{r_{n}\right\}$ are sequences which satisfy the following conditions:

(C1) $\left\{t_{n}\right\} \subset(0,1)$ and $\lim _{n \rightarrow \infty} t_{n}=0$;

(C2) $\left\{r_{n}\right\} \subset(0,1)$ and $\liminf _{n \rightarrow \infty} r_{n}>0$.

Then, the sequence $\left\{x_{n}\right\}$ converges strongly to $P_{F} x_{0}$. 
Proof. First, we rewrite the algorithm (4.1) as the following:

$$
\left\{\begin{array}{l}
x_{0} \in C, D_{0}=C, \\
C_{n}=\overline{c o}\left\{z \in C:\left\|z-T_{n} z\right\| \leq t_{n}\left\|x_{n}-T_{n} x_{n}\right\|\right\}, \quad n \geq 1, \\
D_{n}=\left\{z \in D_{n-1}:\left\langle S_{r_{n}} x_{n}-z, J\left(x_{n}-S_{r_{n}} x_{n}\right)\right\rangle \geq 0\right\}, n \geq 1, \\
x_{n+1}=P_{C_{n} \cap D_{n}} x_{0}, n \geq 0,
\end{array}\right.
$$

where $S_{r}$ is the mapping defined by (3.1) for all $r>0$. We first show that the sequence $\left\{x_{n}\right\}$ is well defined. It is easy to verify that $C_{n} \cap D_{n}$ is closed and convex and $\Omega \subset C_{n}$ for all $n \geq 0$. Next, we prove that $\Omega \subset C_{n} \cap D_{n}$. Since $D_{0}=C$, we also have $\Omega \subset C_{0} \cap D_{0}$. Suppose that $\Omega \subset C_{k-1} \cap D_{k-1}$ for $k \geq 2$. It follows from Lemma (3) that

$$
\left\langle S_{r_{k}} x_{k}-S_{r_{k}} u, J\left(S_{r_{k}} u-u\right)-J\left(S_{r_{k}} x_{k}-x_{k}\right)\right\rangle \geq 0,
$$

for all $u \in \Omega$. This implies that

$$
\left\langle S_{r_{k}} x_{k}-u, J\left(x_{k}-S_{r_{k}} x_{k}\right)\right\rangle \geq 0,
$$

for all $u \in \Omega$. Hence, $\Omega \subset D_{k}$. By the mathematical induction, we get that $\Omega \subset C_{n} \cap$ $D_{n}$ for each $n \geq 0$ and hence $\left\{x_{n}\right\}$ is well defined. Let $w=P_{F} x_{0}$. Since $\Omega \subset C_{n} \cap D_{n}$ and $x_{n+1}=P_{C_{n} \cap D_{n}} x_{0}$, we have

$$
\left\|x_{n+1}-x_{0}\right\| \leq\left\|w-x_{0}\right\|, \quad n \geq 0 .
$$

Since $\left\{x_{n}\right\}$ is bounded, there exists a subsequence $\left\{x_{n_{i}}\right\}$ of $\left\{x_{n}\right\}$ such that $x_{n_{i}} \rightarrow v \in C$. Since $x_{n+2} \in D_{n+1} \subset D_{n}$ and $x_{n+1}=P_{C_{n} \cap D_{n}} x_{0}$, we have

$$
\left\|x_{n+1}-x_{0}\right\| \leq\left\|x_{n+2}-x_{0}\right\| .
$$

Since $\left\{x_{n}-x_{0}\right\}$ is bounded, we have $\lim _{n \rightarrow \infty}|| x_{n}-x_{0} \|=d$ for some a constant $d$. Moreover, by the convexity of $D_{n}$, we also have $\frac{1}{2}\left(x_{n+1}+x_{n+2}\right) \in D_{n}$ and hence

$$
\left\|x_{0}-x_{n+1}\right\| \leq\left\|x_{0}-\frac{x_{n+1}+x_{n+2}}{2}\right\| \leq \frac{1}{2}\left(\left\|x_{0}-x_{n+1}\right\|+\left\|x_{0}-x_{n+2}\right\|\right) .
$$

This implies that

$$
\lim _{n \rightarrow \infty}\left\|\frac{1}{2}\left(x_{0}-x_{n+1}\right)+\frac{1}{2}\left(x_{0}-x_{n+2}\right)\right\|=\lim _{n \rightarrow \infty}\left\|x_{0}-\frac{x_{n+1}+x_{n+2}}{2}\right\|=d .
$$

By Lemma 2.1, we have $\lim _{n \rightarrow \infty}|| x_{n}-x_{n+1} \|=0$.

Next, we show that $v \in \bigcap_{n=0}^{\infty} F\left(T_{n}\right)$. Since $x_{n+1} \in C_{n}$ and $t_{n}>0$, there exists $m \in \mathcal{N}$, $\left\{\lambda_{0}, \lambda_{1}, \ldots, \lambda_{m}\right\} \subset[0,1]$ and $\left\{y_{0}, y_{1}, \ldots, y_{m}\right\} \subset C$ such that

$$
\sum_{i=1}^{m} \lambda_{i}=1,\left\|x_{n+1}-\sum_{i=0}^{m} \lambda_{i} y_{i}\right\|<t_{n} \text {, and }-\left|y_{i}-T_{n} y_{i}\right|\left|\leq t_{n}\right| \mid x_{n}-T_{n} x_{n} \|
$$


for each $i=0,1, \ldots, m$. Since $C$ is bounded, by Lemma 2.2, we have

$$
\begin{aligned}
\left\|x_{n}-T_{n} x_{n}\right\| \leq & \left\|x_{n}-x_{n+1}\right\|+\left\|x_{n+1}-\sum_{i=0}^{m} \lambda_{i} y_{i}\right\|+\left\|\sum_{i=0}^{m} \lambda_{i} y_{i}-\sum_{i=0}^{m} \lambda_{i} T_{n} y_{i}\right\| \\
& +\left\|\sum_{i=0}^{m} \lambda_{i} T_{n} y_{i}-T_{n}\left(\sum_{i=0}^{m} \lambda_{i} y_{i}\right)\right\|+\left\|T_{n}\left(\sum_{i=0}^{m} \lambda_{i} y_{i}\right)-T_{n} x_{n}\right\| \\
\leq & 2\left\|x_{n}-x_{n+1}\right\|+(2+2 M) t_{n} \\
& +\gamma^{-1}\left(\max _{0 \leq i \leq j \leq m}\left(\left\|y_{i}-y_{j}\right\|-\left\|T_{n} y_{i}-T_{n} y_{j}\right\|\right)\right) \\
\leq & 2\left\|x_{n}-x_{n+1}\right\|+(2+2 M) t_{n} \\
& +\gamma^{-1}\left(\max _{0 \leq i \leq j \leq m}\left(\left\|y_{i}-T_{n} y_{i}\right\|-\left\|y_{j}-T_{n} y_{j}\right\|\right)\right) \\
\leq & 2\left\|x_{n}-x_{n+1}\right\|+(2+2 M) t_{n}+\gamma^{-1}\left(4 M t_{n}\right),
\end{aligned}
$$

where $M=\sup _{n \geq 0}|| x_{n}-w \|$. It follows from (C1) that $\lim _{n \rightarrow \infty}|| x_{n}-T_{n} x_{n} \|=0$. Since $\left\{T_{n}\right\}$ satisfies the NST-condition, we have $v \in \bigcap_{n=0}^{\infty} F\left(T_{n}\right)$.

Next, we show that $v \in G E P(f)$. By the construction of $D_{n}$, we see from (2.2) that $S_{r_{n}} x_{n}=P_{D_{n}} x_{n}$. Since $x_{n+1} \in D_{n}$, we obtain

$$
\left\|x_{n}-S_{r_{n}} x_{n}\right\| \leq\left\|x_{n}-x_{n+1}\right\| \rightarrow 0,
$$

as $n \rightarrow \infty$. From (C2), we also have

$$
\frac{1}{r_{n}}\left\|J\left(x_{n}-S_{r_{n}} x_{n}\right)\right\|=\frac{1}{r_{n}}\left\|x_{n}-S_{r_{n}} x_{n}\right\| \rightarrow 0,
$$

as $n \rightarrow \infty$. Since $\left\{x_{n}\right\}$ is bounded, it has a subsequence $\left\{x_{n_{i}}\right\}$ which weakly converges to some $v \in E$.

By (4.4), we also have $S_{r_{n_{i}}} \rightarrow v$. By the definition of $S_{r_{n_{j}}}$, for each $y \in C$, we obtain

$$
f\left(S_{r_{n_{i}}} x_{n_{i}}, y\right)+\left\langle A S_{r_{n_{i}}} x_{n_{i}}, y-S_{r_{n_{i}}} x_{n_{i}}\right\rangle+\frac{1}{r_{n_{i}}}\left\langle y-S_{r_{n_{i}}} x_{n_{i}} J\left(S_{r_{n_{i}}} x_{n_{i}}-x_{n_{i}}\right)\right\rangle \geq 0 .
$$

By (A3) and (4.4), we have

$$
f(v, y)+\langle A v, y-v\rangle \geq 0, \quad \forall y \in C .
$$

This shows that $v \in G E P(f)$ and hence $v \in \Omega:=\bigcap_{n=0}^{\infty} F\left(T_{n}\right) \cap G E P(f)$.

Note that $w=P_{\Omega} x_{0}$. Finally, we show that $x_{n} \rightarrow w$ as $n \rightarrow \infty$. By the weakly lower semicontinuity of the norm, it follows from (4.3) that

$$
\left\|x_{0}-w\right\| \leq\left\|x_{0}-v\right\| \leq \liminf _{i \rightarrow \infty}\left\|x_{0}-x_{n_{i}}\right\| \leq \limsup _{i \rightarrow \infty}\left\|x_{0}-x_{n_{i}}\right\| \leq\left\|x_{0}-w\right\| .
$$

This shows that

$$
\lim _{i \rightarrow \infty}\left\|x_{0}-x_{n_{i}}\right\|=\left\|x_{0}-w\right\|=\left\|x_{0}-v\right\|
$$

and $v=w$. Since $\mathrm{E}$ is uniformly convex, we obtain that $x_{0}-x_{n_{i}} \rightarrow x_{0}-w$. It follows that $x_{n_{i}} \rightarrow w$. Hence, we have $x_{n} \rightarrow w$ as $n \rightarrow w$. This completes the proof.

\section{Deduced theorems}

If we take $f \equiv 0$ and $A \equiv 0$ in Theorem 4.1, then we obtain the following result. 
Theorem 5.1. Let $E$ be a uniformly convex and smooth Banach space, $C$ a nonempty, bounded, closed, and convex subset of $E$ and $\left\{T_{n}\right\}_{n=0}^{\infty} a$ sequence of nonexpansive mappings of $C$ into itself such that $\Omega:=\bigcap_{n=0}^{\infty} F\left(T_{n}\right) \neq$ Dand suppose that $\left\{T_{n}\right\}_{n=0}^{\infty}$ satisfies the NST-condition. Let $\left\{x_{n}\right\}$ be the sequence in C generated by

$$
\left\{\begin{array}{l}
x_{0} \in C, D_{0}=C, \\
C_{n}=\overline{c o}\left\{z \in C:\left\|z-T_{n} z\right\| \leq t_{n}\left\|x_{n}-T_{n} x_{n}\right\|\right\}, n \geq 1, \\
x_{n+1}=P_{C_{n}} x_{0}, n \geq 0 .
\end{array}\right.
$$

If $\left\{t_{n}\right\} \subset(0,1)$ and $\lim _{n \rightarrow \infty} t_{n}=0$, then $\left\{x_{n}\right\}$ converges strongly to $P_{\Omega^{2}} x_{0}$.

Remark 5.2. By Lemma 2.3, if we define $T_{n}=\alpha_{n} I+\left(1-\alpha_{n}\right) \sum_{k=1}^{n} \beta_{n}^{k} S_{k}$ for all $n \geq 0$ in Theorems 3.1 and 5.1, then the theorems also hold.

If we take $T_{n} \equiv I$, the identity mapping on $C$, for all $n \geq 0$ in Theorem 4.1, then we obtain the following result.

Theorem 5.3. Let $E$ be a uniformly convex and smooth Banach space, $C$ a nonempty, bounded, closed, and convex subset of E. Let $f$ be a bifunction from $C \times C$ to $\mathcal{R}$ satisfying (A1)-(A4) and $A$ an $\alpha$-inverse strongly monotone mapping of $C$ into $E^{*}$. Let $\left\{x_{n}\right\}$ be the sequence in $C$ generated by

$$
\left\{\begin{array}{l}
x_{0} \in C, D_{0}=C \\
u_{n} \in C \text { such that } f\left(u_{n}, y\right)+\left\langle A u_{n}, y-u_{n}\right\rangle+\frac{1}{r_{n}}\left\langle y-u_{n}, J\left(u_{n}-x_{n}\right)\right\rangle \geq 0, \forall y \in C, n \geq 0, \\
D_{n}=\left\{z \in D_{n-1}:\left\langle u_{n}-z, J\left(x_{n}-u_{n}\right)\right\rangle \geq 0\right\}, \quad n \geq 1, \\
x_{n+1}=P_{D_{n}} x_{0}, \quad n \geq 0 .
\end{array}\right.
$$

If $\left\{r_{n}\right\} \subset(0,1)$ and $\lim _{\inf _{n \rightarrow \infty}} r_{n}>0$, then $\left\{x_{n}\right\}$ converges strongly to $P_{G E P}(f) x_{0}$.

If we take $A \equiv 0$ in Theorem 4.1, then we obtain the following result concerning an equilibrium problem in a Banach space setting.

Theorem 5.4. Let $E$ be a uniformly convex and smooth Banach space and $C$ be a nonempty, bounded, closed, and convex subset of $E$. Let $f$ be a bifunction from $C \times C$ to Rsatisfying (A1)-(A4) and let $\left\{T_{n}\right\}_{n=0}^{\infty}$ be a sequence of nonexpansive mappings of $C$ into itself such that $\Omega:=\bigcap_{n=0}^{\infty} F\left(T_{n}\right) \cap E P(f) \neq$ Øand suppose that $\left\{T_{n}\right\}_{n=0}^{\infty}$ satisfies the NSTcondition. Let $\left\{x_{n}\right\}$ be the sequence in $C$ generated by

$$
\left\{\begin{array}{l}
x_{0} \in C, D_{0}=C, \\
C_{n}=\overline{c o}\left\{z \in C:\left\|z-T_{n} z\right\| \leq t_{n}\left\|x_{n}-T_{n} x_{n}\right\|\right\}, \quad n \geq 1, \\
u_{n} \in C \text { such that } f\left(u_{n}, y\right)+\frac{1}{r_{n}}\left\langle y-u_{n}, J\left(u_{n}-x_{n}\right)\right\rangle \geq 0, \quad \forall y \in C, \quad n \geq 0, \\
D_{n}=\left\{z \in D_{n-1}:\left\langle u_{n}-z, J\left(x_{n}-u_{n}\right)\right\rangle \geq 0\right\}, \quad n \geq 1, \\
x_{n+1}=P_{C_{n} \cap D_{n}} x_{0}, \quad n \geq 0,
\end{array}\right.
$$

where $\left\{t_{n}\right\}$ and $\left\{r_{n}\right\}$ are sequences which satisfy the conditions:

(C1) $\left\{t_{n}\right\} \subset(0,1)$ and $\lim _{n \rightarrow \infty} t_{n}=0$;

(C2) $\left\{r_{n}\right\} \subset(0,1)$ and $\liminf _{n \rightarrow \infty} r_{n}>0$.

Then, the sequence $\left\{x_{n}\right\}$ converges strongly to $P_{\Omega} x_{0}$. 
GEP: generalized equilibrium problem.

\section{Acknowledgements}

U. Kamraksa was supported by grant from under the program "Strategic Scholarships for Frontier Research Network for the Ph.D." Program Thai Doctoral degree from the Office of the Higher Education Commission, Thailand. The project was supported by the "Centre of Excellence in Mathematics" under the Commission on Higher Education, Ministry of Education, Thailand and the grant from under the program Strategic Scholarships for Frontier Research Network for the Ph.D. Program Thai Doctoral degree from the Office of the Higher Education Commission.

\section{Author details}

'Department of Mathematics, Faculty of Science, Naresuan University, Phitsanulok 65000, Thailand ${ }^{2}$ Centre of Excellence in Mathematics, Che, Si Ayutthaya Road, Bangkok 10400, Thailand

Received: 26 December 2010 Accepted: 28 June 2011 Published: 28 June 2011

\section{References}

1. Takahashi, S, Takahashi, W: Strong convergence theorem for a generalized equilibrium problem and a nonexpansive mappings in a Hilbert space. J Nonlinear Anal. 69, 1025-1033 (2008). doi:10.1016/..na.2008.02.042

2. Kohsaka, F, Takahashi, W: Strong convergence of an iterative sequence for maximal monotone operators in a Banach space. Abstr Appl Anal. 3, 239-249 (2004)

3. Martinet, B: Regularisation d'inequations variationnelles par approximations successives. Rev Fr Autom Inf Res Oper. 4, 154-159 (1970)

4. Mann, WR: Mean value methods in iteration. Proc Am Math Soc. 4, 506-510 (1953). doi:10.1090/S0002-9939-19530054846-3

5. Bauschke, $\mathrm{HH}$, Matouskova, E, Reich, S: Projection and proximal point methods: convergence results and counterexamples. Nonlinear Anal. 56, 715-738 (2004). doi:10.1016/j.na.2003.10.010

6. Genal, A, Lindenstrass, J: An example concerning fixed points. Isr J Math. 22, 81-86 (1975). doi:10.1007/BF02757276

7. Reich, S: Weak convergence theorems for nonexpansive mappings in Banach spaces. J Math Anal Appl. 67, 274-276 (1979). doi:10.1016/0022-247X(79)90024-6

8. Bauschke, HH, Combettes, PL: A weak-to-strong convergence principle for Fejer-Monotone methods in Hilbert spaces. Math Oper Res. 26, 248-264 (2001). doi:10.1287/moor.26.2.248.10558

9. Nakajo, K, Shimoji, K, Takahashi, W: Strong convergence to common fixed points of families of nonexpansive mappings in Banach spaces. J Nonlinear Convex Anal. 8, 11-34 (2007)

10. Nakajo, K, Shimoji, K, Takahashi, W: Strong convergence theorems by the hybrid method for families of nonexpansive mappings in Hilbert spaces. Taiwan J Math. 10, 339-360 (2006)

11. Takahashi, W, Takeuchi, Y, Kubota, R: Strong convergence theorems by hybrid methods for families of nonexpansive mappings in Hilbert spaces. J Math Anal Appl. 341, 276-286 (2008). doi:10.1016/j.jmaa.2007.09.062

12. Nakajo, K, Takahashi, W: Strong convergence theorems for nonexpansive mappings and nonexpansive semigroups. J Math Anal Appl. 279, 372-379 (2003). doi:10.1016/S0022-247X(02)00458-4

13. $\mathrm{Xu}, \mathrm{H}-\mathrm{K}$ : Strong convergence of approximating fixed point sequences for nonexpansive mappings. Bull Aust Math Soc. 74, 143-151 (2006). doi:10.1017/\$0004972700047535

14. Matsushita, S, Takahashi, W: Approximating fixed points of nonexpansive mappings in a Banach space by metric projections. Appl Math Comput. 196, 422-425 (2008). doi:10.1016/j.amc.2007.06.006

15. Kimura, Y, Nakajo, K: Some characterizations for a family of nonexpansive mappings and convergence of a generated sequence to their common fixed point. Fixed Point Theory Appl. (2010)

16. Takahashi, W: Nonlinear Functional Analysis. Yokohama Publishers (2000)

17. Zeidler, E: Nonlinear Functional Analysis and Its Applications, vol. I: Fixed-Point Theorems. Springer, New York (1986)

18. Bruck, RE: On the convex approximation property and the asymptotic behaviour of nonlinear contractions in Banach spaces. Isr J Math. 38, 304-314 (1981). doi:10.1007/BF02762776

19. Bruck, RE: Properties of fixed-point sets of nonexpansive mappings in Banach spaces. Trans Am Math Soc. 179, 251-262 (1973)

20. Aoyama, K, Kimura, Y, Takahashi, W, Toyoda, M: Approximation of common fixed points of a countable family of nonexpansive mappings in a Banach space. Nonlinear Anal. 67, 2350-2360 (2007). doi:10.1016/j.na.2006.08.032

21. Fan, K: A generalization of Tychonoffs fixed point theorem. Math Ann. 142, 305-310 (1961). doi:10.1007/BF01353421

22. Takahashi, W, Zembayashi, K: Strong and weak convergence theorems for equilibrium problems and relatively nonexpansive mappings in Banach spaces. J Nonlinear Anal. 70, $45-57$ (2009). doi:10.1016/j.na.2007.11.031

23. Ceng, L-C, Yao, J-C: A hybrid iterative scheme for mixed equilibrium problems and fixed point problems. J Comput Appl Math. 214, 186-201 (2008). doi:10.1016/j.cam.2007.02.022

doi:10.1186/1687-1812-2011-11

Cite this article as: Kamraksa and Wangkeeree: Existence and iterative approximation for generalized equilibrium problems for a countable family of nonexpansive mappings in banach spaces. Fixed Point Theory and Applications 2011 2011:11. 